\title{
AF classification and ablation strategy: time for a new paradigm
}

Submitted: 17 May 2016; Accepted: 30 May 2016; Published online: 04 June 2016

Catheter ablation for symptomatic atrial fibrillation has emerged as a successful therapy for patients with medically refractive atrial fibrillation. Nearly uniform superior short- and long-term success rates compared with antiarrhythmic medications have prompted recent guideline changes that suggest ablation can be considered before use of pharmacologic therapies [1]. Traditionally, ablation strategies for AF have been divided into either pulmonary vein isolation (PVI) alone or PVI and substrate ablation. The trigger alone strategy has been the primary strategy in patients with paroxysmal atrial fibrillation, with a recent clinical trial reporting greater than a 70\% 12 month success rate with the use of contact force sensing technology [2]. Those with persistent and long-standing persistent atrial fibrillation or moderate- severe structural heart disease continue to experience suboptimal results and often require multiple ablations [1]. Many operators believe that in these patients additional ablation beyond PVI is needed. Additional ablation approaches have included such strategies as linear ablation, ablation of complex fractionated electrograms (CFAE), focal impulse or rotor modulation (FIRM), among others. However, recent results of the multi-center STAR-AF 2 trial have shown that linear ablation and ablation of CFAE have not been superior to that of PVI alone in persistent AF [3]. As such, the ideal ablation strategy in these patients uncertain. Furthermore, there appears to be a subset of patients with paroxysmal AF that have poor long term outcomes from PVI alone, as well as a subset of patients with persistent $\mathrm{AF}$ patients that have excellent outcomes from PVI alone [4]. Given these data, we believe that classifying $\mathrm{AF}$ as either paroxysmal or persistent is inadequate to guide ablation strategy.

More recently, we, as well as other centers, have begun focusing on the presence of atrial fibrosis in the left atrium. Atrial fibrosis and scar have been established as important cellular substrates for AF [5]. Furthermore, atrial fibrosis and scar may serve as sources of micro and macro reentry, anchoring of rotors, and rapid drivers [3]. A recent study showed that atrial scar burden predicts recurrence of $\mathrm{AF}$ following catheter ablation [6]. In another recent study by McCann et al. [7], late gadolinium enhancement MRI was performed in 386 patients prior to undergoing their first ablation for AF. In the 123 patients with failed ablations, extent of late gadolinium enhancement correlated more with $\mathrm{AF}$ recurrence, rather than the presence of persistent AF. As such, regional fibrosis and scar have been promoted as sources of atrial fibrillation arrhythmia maintenance. Furthermore, a number of groups have demonstrated that the use of electroanatomically guided voltage mapping enables in vivo assessment of fibrosis in the atria of patients with AF [8-10]. Based on these data, we reasoned that incorporating fibrosis and isolating areas of low voltage may be a more efficient ablation strategy than one based solely on either a persistent or paroxysmal AF classification.

In June 2011, we began generating voltage maps of the posterior wall of the LA during sinus rhythm to guide ablation beyond PVI
Nikhil Panda, William Lewis \& Ohad Ziv*

Department of Clinical Cardiac Electrophysiology, Metro Health Atrial Fibrillation Center, USA

*Author for correspondence:

Tel.: +12167782328

oziv@metrohealth.org 
alone in patients classified as persistent AF patients. We used $\mathrm{a}<0.5 \mathrm{mV}$ threshold for abnormally low voltage and focused on the posterior wall since the posterior wall has been targeted for ablation in other studies and has been reported to commonly harbor regions of low voltage bipolar electro grams [6,11]. This shift was an empiric one and only 3 of our 6 operators made the change in ablation strategy. Therefore, 3 of our operators continued to use a "standard" strategy of PVI with additional lesions based on clinical factors- not voltage data.

We recently published a comparison of 1 year outcomes using these two ablation strategies at our institution [12]. At one year post ablation, $80 \%$ of patients in the voltage guided ablation strategy and $57 \%$ of patients in the standard ablation group remained in sinus rhythm $(\mathrm{p}=0.005)$ (Figure 1). Of the 76 patients in the standard ablation group, 43 patients received empiric posterior wall ablation in addition to PVI. Of the 65 patients in the voltage guided ablation group, 27 patients were found to have low voltage in the posterior

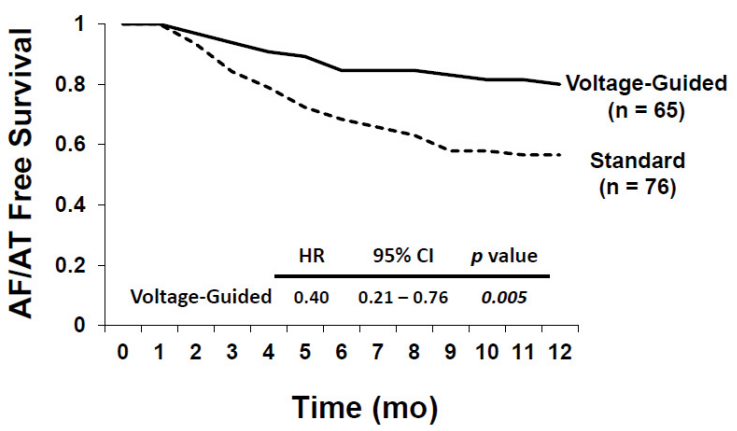

Figure 1: Voltage-guided Ablation Improves 1-year ArrhythmiaFree Survival. 1-year Kaplan Meier arrhythmia-free survival curves comparing VGA vs SA.

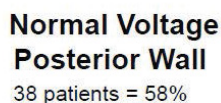

38 patients $=58 \%$

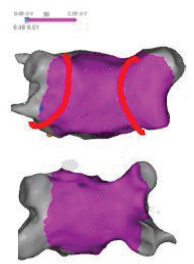

Pulmonary Vein Isolation
Low Voltage Posterior Wall 27 patients $=42 \%$

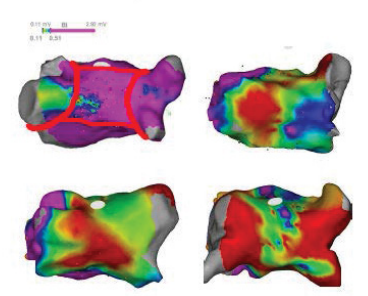

Pulmonary Vein Isolation

Posterior Wall Isolation
Figure 2: Voltage-guided Ablation. Shown are examples of posterior wall mapping performed during VGA. Panel A shows two examples of normal voltage left atria. These patients received only PVAI. Panel B shows four examples of posterior wall maps demonstrating progressively increased scar burden. These patients received both PVAl and posterior wall isolation.

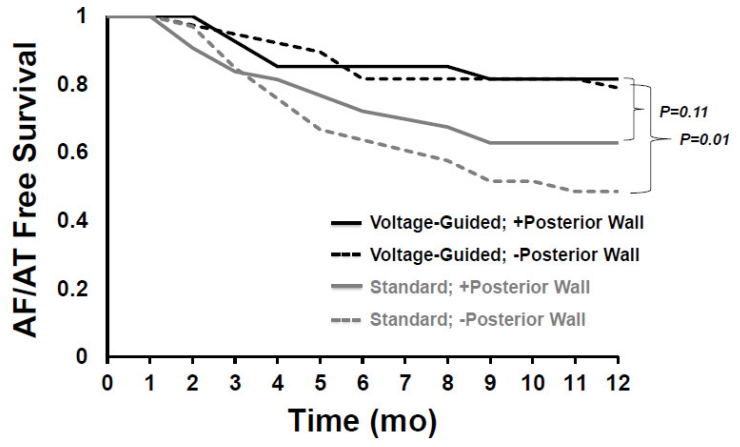

Figure 3: Effect of Posterior Wall Ablation. Sub-group analysis showing 1-year Kaplan Meier arrhythmia-free survival curves in VGA and SA groups with or without posterior wall ablation.

wall and thus posterior wall isolation (Figure 2). In a subgroup analysis, we found that empirically isolating the posterior wall without data on voltage or limiting ablation to PVI alone without data on voltage $(63 \%$ vs $48 \%$, respectively) yielded inferior results to guided ablation of the posterior wall beyond PVI based on the presence of low voltage (AF/AT free survival at 1 year of $81 \%$ with posterior wall ablation vs $79 \%$ without posterior wall ablation) (Figure 3). Further analysis into the type of atrial arrhythmias that recurred showed that the voltage guided strategy reduced AF in patients receiving no posterior wall ablation and reduced atrial flutter or atrial tachycardia recurrences in patients receiving posterior wall ablation (Figure 4). Our study established that in patients with persistent $\mathrm{AF}$, utilizing the presence or absence of posterior wall low voltage to guide posterior wall ablation beyond PVI significantly reduces 1-year AF/AT recurrence when compared to a non-voltage guided ablation approach. Interestingly, our study showed that the mere presence of posterior wall ablation was not a predictor of long term outcomes, but that further ablation should be guided by the presence of low voltage. Conversely, persistent AF patients without evidence of left atrial low voltage may be sufficiently treated with PVI alone, as half the patients in the voltage guided ablation group did not have posterior wall low voltage and thus received only PVI. Thus, voltage guided ablation strategy appears to pre-select patient with persistent $\mathrm{AF}$ who would benefit from PVI alone or those who would benefit from PVI and posterior wall isolation.

The use of voltage to guide further ablation does not appear limited to persistent AF patients. Kottkamp et. al. (Kottkamp, JCE, 2015) recently published a study where they isolated areas of left atrial fibrosis following voltage mapping of the left atrium in paroxysmal AF in patients undergoing redo ablation, excluding those that had pulmonary vein reconnection. Low voltage was identified and isolated in all 10 paroxysmal AF 


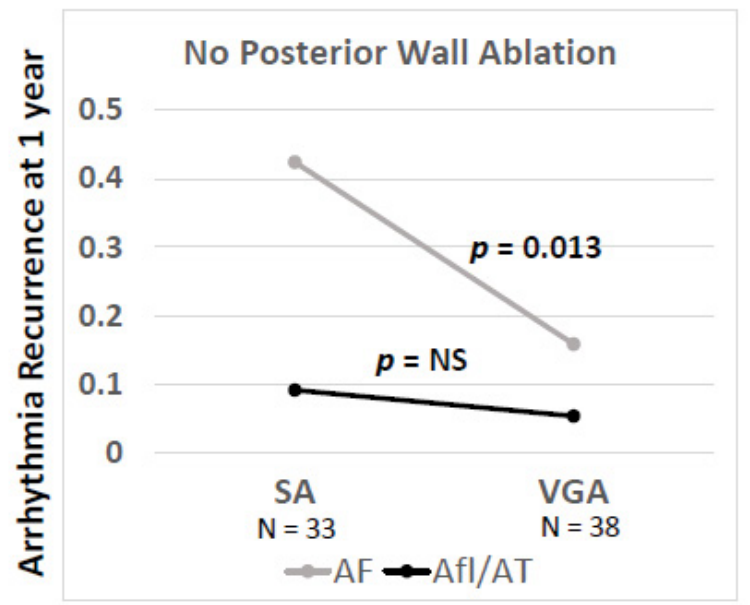

Panel A

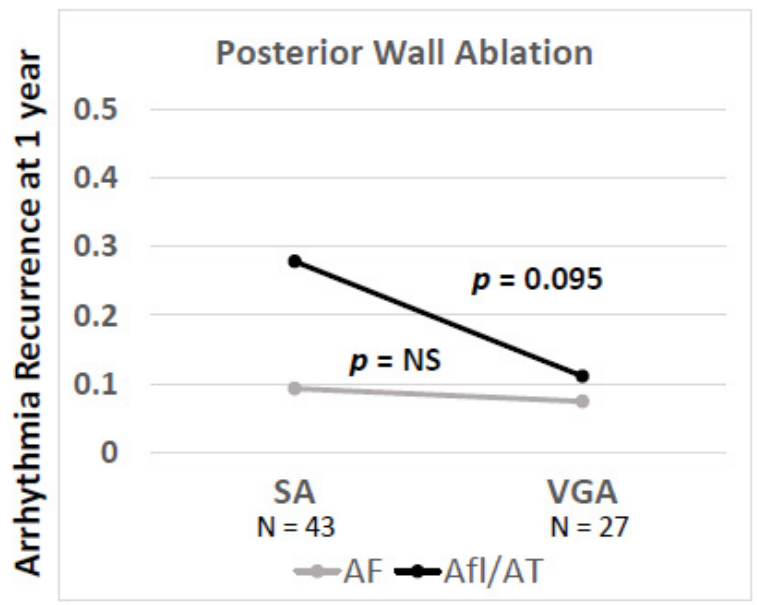

Panel B

Figure 4: Effect of strategy on recurrence by type of arrhythmia. Subgroup analysis showing recurrence rates of AF or Afl/ ATrecurrence rates in patients receiving posterior wall ablation or no posterior wall ablation in either VGA or SA groups. VGA group had lower AF recurrence when no posterior wall ablation was performed (panel A) and lower Afl/AT recurrences when posterior wall was performed compared to SA group (panel B).

patients undergoing re-do ablation. During a mean follow-up of 20 months, 9/10 patients remained free of AF. In support of our data, the Kottkamp study also identified areas of low voltage in persistent AF patients undergoing first time ablation. Out of 31 patients, low voltage was identified in 18 patients and isolated in 17 , as one patient had fibrosis covering the entire left atrium. In 13 patients, low voltage was not identified and only PVI was performed. Following a mean followup of 12.5 months, $22 / 31$ of all patients $(71 \%)$ and $15 / 18$ patients $(83 \%)$ in the persistent $\mathrm{AF}$ group remained free of atrial arrhythmias following a single procedure.

Both our study and that of Kottkamp et al. [13] suggest that obtaining a voltage map of all patients undergoing $\mathrm{AF}$ ablation may identify those that would do well with PVI alone, or those that may need additional ablation, such as isolating areas of low voltage. Importantly, the classification of persistent or paroxysmal AF based on duration of AF episodes does not appear to be a strong predictor of low voltage in the LA. In fact, in our data set of patients with persistent $\mathrm{AF}$, the presence of posterior wall low voltage did not correlate well with duration of AF episodes. While the average longest $\mathrm{AF}$ episode was 104 days in patients with low voltage and 81 days with patients without low voltage, this difference was not statistically significant.
Furthermore, while LA diameter was significantly larger in patients with low voltage $(4.6 \mathrm{~cm}$ compared to $4.2 \mathrm{~cm} \mathrm{p}=0.04$ ), in a multivariate analysis, only three independent predictors of low voltage emerged: heart failure, diabetes and female gender. Thus, the criteria that we have been using to target ablation beyond PVI do not appear to be valid.

We propose a new classification of $\mathrm{AF}$ to better guide ablation strategy: $\mathrm{AF}$ in the presence of atrial fibrosis (Fibrotic AF: FAF) and AF in the absence of atrial fibrosis (Non-fibrotic AF: nFAF). This classification allows for a new paradigm in selecting an individually tailored ablation approach that may be unique to each patient based on the presence and extent of atrial fibrosis, rather than simply the duration of $\mathrm{AF}$ episodes as with paroxysmal or persistent AF. Despite the promise that voltage mapping appears to hold, significant further investigation is needed. Larger, multicenter randomized trials are needed to corroborate these early data. We need further understanding of potential mechanistic differences in $\mathrm{AF}$ with and without fibrosis. The ideal mechanism for identifying fibrosis needs to be assessed (late gadolinium enhancement on MRI versus bipolar voltage mapping). Still, we believe that classifying AF based on presence of fibrosis will have great practical value and will help guide better guide ablation strategy. 


\section{References}

1. Calkins H. Hybrid thoracoscopic and transvenous catheter ablation of atrial fibrillation: is this the answer we are searching for? J. Am. Coll. Cardiol. 60, 62-63 (2012).

2. Natale A, Reddy VY, Monir G, et al. Paroxysmal AF catheter ablation with a contact force sensing catheter: results of the prospective, multicenter SMART-AF trial. J. Am. Coll. Cardiol. 64, 647-656 (2014).

3. Verma A, Jiang CY, Betts TR, et al. Approaches to catheter ablation for persistent atrial fibrillation. N. Engl. J. Med. 372, 1812-1822 (2015).

4. Rostock T, Salukhe TV, Steven D, et al. Long-term singleand multiple-procedure outcome and predictors of success after catheter ablation for persistent atrial fibrillation. Heart. Rhythm. 8, 1391-1397 (2011).

5. Burstein B, Nattel S. Atrial fibrosis: mechanisms and clinical relevance in atrial fibrillation. J. Am. Coll. Cardiol. 51, 802809 (2008).

6. Pump A, Di Biase L, Price J, et al. Efficacy of catheter ablation in nonparoxysmal atrial fibrillation patients with severe enlarged left atrium and its impact on left atrial structural remodeling. J. Cardiovasc. Electrophysio. 24, 1224-1231 (2013).

7. McGann C, Akoum N, Patel A, et al. Atrial fibrillation ablation outcome is predicted by left atrial remodeling on MRI. Circ. Arrhythm. Electrophysiol. 7, 23-30 (2014).
8. Spragg DD, Khurram I, Zimmerman SL, et al. Initial experience with magnetic resonance imaging of atrial scar and co-registration with electroanatomic voltage mapping during atrial fibrillation: success and limitations. Heart. Rhythm . 9, 2003-2009 (2012).

9. Marcus GM, Yang Y, Varosy PD, Ordovas K, Tseng ZH, et al. (2007) Regional left atrial voltage in patients with atrial fibrillation. Heart Rhythm 4: 138-144.

10. Squara F, Frankel DS, Schaller R, et al. Voltage mapping for delineating inexcitable dense scar in patients undergoing atrial fibrillation ablation: a new end point for enhancing pulmonary vein isolation. Heart. Rhythm. 11, 1904-1911 (2014).

11. Kumagai K, Sakamoto T, Nakamura K, et al. Combined dominant frequency and complex fractionated atrial electrogram ablation after circumferential pulmonary vein isolation of atrial fibrillation. J. Cardiovasc. Electrophysiol. 24, 975-983 (2013).

12. Cutler MJ, Johnson J, Abozguia K, Impact of Voltage Mapping to Guide Whether to Perform Ablation of the Posterior Wall in Patients With Persistent Atrial Fibrillation. J. Cardiovasc. Electrophysiol. 27: 13-21 (2016).

13. Kottkamp H, Berg J, Bender R, Rieger A, et al. Schreiber D Box Isolation of Fibrotic Areas (BIFA): A Patient-Tailored Substrate Modification Approach for Ablation of Atrial Fibrillation. J. Cardiovasc. Electrophysiol. 27, 22-30 (2016). 